\title{
SOME ELEMENTARY ASPECTS OF MODULAR FUNCTIONS IN SEVERAL VARIABLES
}

\author{
HARVEY COHN ${ }^{1}$
}

1. Introduction. We shall speak of "elementary" aspects as those which are understood by direct analogy with the rational, classical, "well-known" one-dimensional modular functions, namely, Eisenstein series and theta-functions arising through number-theory. By contrast, some "nonelementary" aspects are those arising in the generalization process in the Siegel theory of modular functions. Actually, some of the problems of current interest are more topological than analytic or number theoretic and in some special instances, functions arising in number theory provide illustrations ${ }^{2}$ of topological results (see $\$ 16$ below), such as compactification parameters.

The basic classical theory [16] can be built around the number of decompositions of an integer into the sum of $t$-squares (we take $t \equiv 0(\bmod 4))$. The number of decomposition depends on functions like $\sigma_{t / 2-1}(m)$ the sum of the $(t / 2-1)$ powers of the divisors of $m$ by a set of formulas (in $\$ 11$ below) which are now "classical."

A series of papers of Siegel, Götzky [9], Maass [14], Gundlach [11], etc., has extended this study to the field $Q(\sqrt{ } 5)$. It turns out, however, that a more direct, almost a "word-for-word" analogy can be constructed using $Q(\sqrt{ } 2), Q(\sqrt{ } 3)$ as shown in recent papers [1], [3], [5]. This study is overshadowed, however, by impending difficulties. The fundamental domain involved in $Q(\sqrt{ } 2)$ or $Q(\sqrt{ } 3)$ is actually of a more difficult topological nature than that of $Q(\sqrt{ } 5)$ as shown (see $\$ 9$ below) by electronic computer results [6], [7]. This difficulty must come to the surface eventually.

At the same time that number theoretic formulas have an incredibly easy generalization by quadratic modular functions, we encounter the difficulty that such functions are not defined on analytic manifolds except in a few artificial cases (as noticed by Gundlach [10]). Thus classical arguments have not traditionally been extended from

An address delivered before the Palo Alto meeting of the Society on April 24, 1965, by invitation of the Committee to Select Hour Speakers for Western Sectional Meetings; received by the editors June 7, 1965.

1 Supported by NSF G-7412. Computer support donated by the Argonne National Laboratories of the USAEC with cooperation of Dr. Wallace J. Givens of the Applied Mathematics Division.

2 Such results were first announced in tentative form as a short communication to the 1962 International Congress at Stockholm. 
one variable to two variables by the use of algebraic function theory, only by a more mechanical "method of descent" (see $\$ 14$ below). We therefore seek to examine an elementary case where such improvement might still be possible (in $\$ 16$ below).

\section{The ClAssical MODULAR SUBDIVISION}

2. The rational fundamental domain. The classical theory of modular functions of one variable seems to be derived from work of Gauss [8] in potential theory dating back to 1794 (when Gauss was 17 years old!). The modular group $G$ consists of the transformations on the upper half $z$-plane $U$

$$
G: z \rightarrow g(z)=(a z+b) /(c z+d), \quad a d-b c=1
$$

where $a, b, c, d$ are integers. We seek to define a fundamental domain; namely, a subset of the upper half $z$-plane with representatives of each equivalence class of points under $G$.

A very obvious subgroup of $G$ is

$$
G_{\infty}: g(z)=z+b
$$

consisting of all transformations which leave $z=\infty$ invariant. They have as fundamental domain the strip

$$
D_{\infty}:-\frac{1}{2}<\operatorname{Re} z \leqq \frac{1}{2} \quad(\operatorname{Im} z>0) .
$$

It can be seen that $G_{\infty}$ together with $g \rightarrow-1 / z$ determines $G$, or that $\{z \rightarrow z+1, z \rightarrow-1 / z\}=G$ using the $\{\cdots\}$ for generators. This is a consequence of the euclidean algorithm which can be extended directly to other fields; i.e.,

$$
\left(\begin{array}{ll}
a & b \\
c & d
\end{array}\right)\left(\begin{array}{ll}
1 & n \\
0 & 1
\end{array}\right)=\left(\begin{array}{ll}
a & b+n a \\
c & d+n c
\end{array}\right)
$$

so that the maximum matrix element can be always reduced in size by the use of the generators until a 0 is produced.

For a fixed $z_{0}$ of the upper half plane $U$, consider all transformations $g\left(z_{0}\right)(g \in G)$. Then it is easily seen that for some equivalent point, (i.e., some choice of $g$ ),

$$
\operatorname{Im} g\left(z_{0}\right)=\text { maximum (over choice of } g \in G \text { ). }
$$

For this optimum $g$, then $z_{*}=g\left(z_{0}\right)$ must satisfy

$$
D_{0}:\left|c z_{*}+d\right| \geqq 1 \quad \text { for all }(c, d)=1
$$

(and a region $D_{0}$ is thus defined in $U$ ). This must be so, for otherwise 


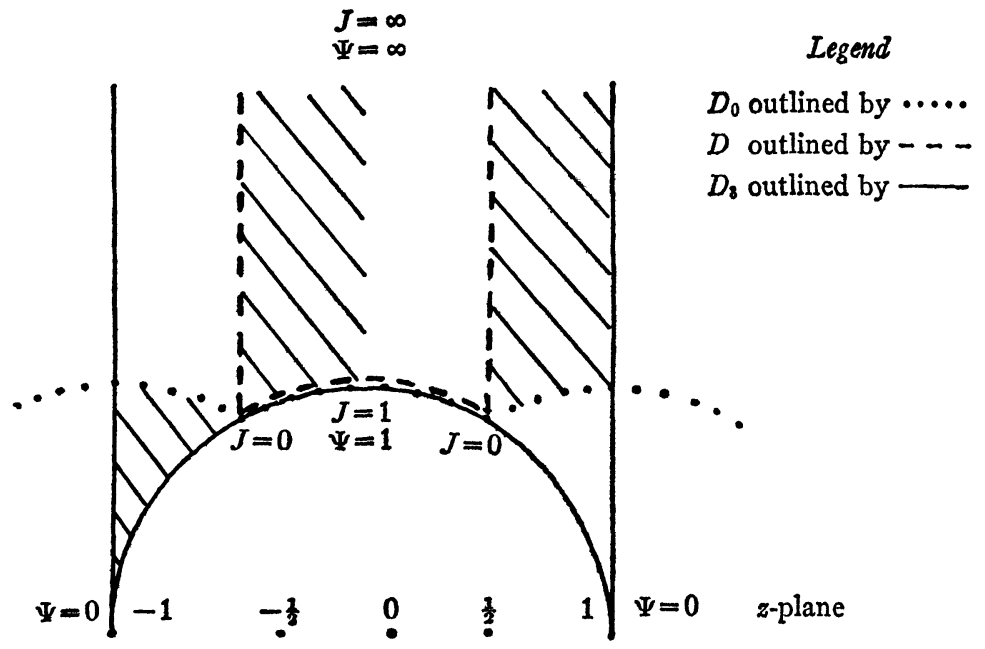

Figure 1. Fundamental domains $D$ for $G$ and $D_{3}$ for $G_{3}$.

a special $g\left(z_{*}\right)=\left(a z_{*}+b\right) /\left(c z_{*}+d\right)$ can be formed for which

$$
\operatorname{Im} g\left(z_{*}\right)=\operatorname{Im} z_{*} /\left|c z_{*}+d\right|^{2}>\operatorname{Im} z_{*} .
$$

It is evident geometrically [6] that (2.6) is implied by $\left|z_{*}+d_{0}\right| \geqq 1$ if $d_{0}$ is the closed integer to $-d / c$ (when $\left.c \neq 0\right)$. Thus (2.6) follows from

$$
\left|z_{*}+d\right| \geqq 1 \quad \text { for all } d ;
$$

and $D_{0}$ consists of the exteriors of unit circles drawn at the integral points of the real axis (intersected with the upper half-plane of course). This is a property which does not extend itself to the case of several variables (see $\$ 9$ below).

The fundamental domain of $G$ (in $U$ ) is then

$$
D=D_{0} \cap D_{\infty}
$$

or

$$
D:\left\{\begin{aligned}
-\frac{1}{2}<\operatorname{Re} z \leqq \frac{1}{2} \\
1 \leqq|z|, \\
0<\operatorname{Im} z
\end{aligned}\right.
$$

with obvious identifications of boundary points. (See the portion of Figure 1 surrounded by a dotted line.)

3. The modular function. At this point we deal with analytic com- 
pactification properties which will prove difficult to generalize to several variables.

First of all, it is trivial to compactify the fundamental domain $D$ (nonanalytically) by adjoining an ideal point $z=\infty$ (often written " $z=i \infty ")$, to obtain a region $\bar{D}$. This presupposes an "ordinary" topology at other points which in turn make special allowance for the matching of boundaries.

Fortunately there is a simple way of introducing the analytic structure on $\bar{D}$ if we use the modular function $J(z)$ which maps $\bar{D}$ onto the $J$-sphere (with $J(\infty)=\infty$ and other points as shown). Thus

$$
\begin{aligned}
J(z) & \approx(z-\rho)^{3} \text { near } z=\rho\left(=\frac{1}{2}[-1+i \sqrt{ } 3]\right), \\
J(z)-1 & \approx(z-i)^{2} \text { near } z=i, \\
J(z) & \approx \exp -2 \pi i z \text { near } z=\infty .
\end{aligned}
$$

Actually the number theoretic properties of $J(z)$ are well-known and need not be explored here. The important thing to note is that at $z=\infty$ (or $J=\infty$ ) the uniformizing parameter of $\bar{D}$ is $1 / J$, or better still,

$$
\zeta=e(2 z)(\approx 1 / J)
$$

where we introduce the convenient designation

$$
e(z)=\exp \pi i z .
$$

We shall generalize this parameter $\zeta$ with "mixed success" later on when we take the case of several variables. For now note that $e(n)=1$ exactly when $n$ is even and $e(n z) \rightarrow 0$ exactly when $n>0$.

From the reflection properties $J(-1 / z)=J(z+1)=J(z)$, hence

$$
J((a z+b) /(c z+d))=J(z)
$$

or, taking "differentials"

$$
J^{\prime}((a z+b) /(c z+d))(c z+d)^{-2}=J^{\prime}(z) .
$$

4. Use of algebraic manifolds. In accordance with the classic theory, a subgroup of finite index $j$ within $G$ has a fundamental domain a superdomain consisting of $j$ replicas of $D$.

For example, a useful subgroup is $G_{3}$ (of index 3)

$$
G_{3}: g(z) \equiv z \text { or }-1 / z(\bmod 2) \text {. }
$$

(The congruence means merely congruences on $a, b, c, d$ in (2.1).) It can be shown that $G_{3}$ is generated by $\{z \rightarrow z+2, z \rightarrow-1 / z\}$. Its fundamental domain is $D_{3}$ shown by the solid lines in Figure 1 . Here $D$ is split into the shaded part where $\operatorname{Im} J>0$ and the unshaded part 
where $\operatorname{Im} J<0$ for convenience in assembling $D_{8}$. Thus,

$$
D_{3}:\left\{\begin{aligned}
-1 & <\operatorname{Re} z \leqq 1, \\
1 & \leqq|z|, \\
0 & <\operatorname{Im} z,
\end{aligned}\right.
$$

with obvious boundary identifications (found by reflections). Then $\bar{D}_{3}$, the compactified region has $z=\infty$ and $z=+1$ added, $(z=-1$ is the same as $z=+1$ since it arises from $z \rightarrow z-2 \equiv z(\bmod 2))$.

By the classical algebraic theory there exists an algebraic function $\psi$ (of $J$ ) which maps $\bar{D}_{8}$ onto the $\psi$-sphere, namely

$$
J(z)=(4 \psi-1)^{8} / 27 \psi
$$

(unique by the specifications, $\psi=0$ at $z=1, \psi=1$ at $z=i, \psi=\infty$ at $z=\infty)$. Strangely enough, algebraic relations like (4.3) abound when we use several variables despite the difficulties in establishing an algebraic structure.

To see the simplest connection with number theory, consider the Eisenstein series summed over all integers $p, q$ not both zero

$$
E_{h}(z)=\sum(p z+q)^{-h}, \quad h \text { even }>2 .
$$

It is easily seen that

$$
E_{h}((a z+b) /(c z+d))=E_{h}(z)(c z+d)^{h} .
$$

Therefore, $E_{h}(z) J^{\prime}(z)^{h / 2}$ is invariant under $G$, hence algebraic (and indeed rational) in $J(z)$. By constructing such functions, we find for instance $E_{4}(z)=$ const. $J^{\prime}(z)^{2} /\{J(z)[J(z)-1]\}$ and

$$
E_{4}^{2}(z)=E_{8}(z) \cdot \text { constant. }
$$

This is a number-theoretic relation since "divisor functions" are involved

$$
E_{h}(z)=2 \zeta(h)+2 \cdot\left[(2 \pi i)^{h} / \Gamma(h)\right] \sum_{1}^{\infty} \sigma_{h-1}(n) e(2 n z)
$$

where

$$
\sigma_{h-1}(n)=\sum_{t \mid n} t^{h-1}
$$

Our typical result leads to the classical identity,

$$
\left[1+240 \sum \sigma_{3}(n) e(2 n z)\right]^{2}=\left[1+480 \sum \sigma_{7}(n) e(2 n z)\right] .
$$

The presence of the divisor function is not hard to generalize to functions of several variables. For the present we shall list several 
rational identities which are easily proved by algebraic arguments and which have starkly simple generalizations to modular functions of two variables.

II. REPRESENTATION BY SQUARES IN RATIONAL INTEGERS

5. The theta-series. We wish to count the number of representations $R_{t}(m)$ of $m$ as the sum of $t$ rational squares. We set

$$
\theta(z)=\sum_{-\infty}^{\infty} e\left(n^{2} z\right) \quad(\operatorname{Im} z>0),
$$

and seek to find the Fourier expansion,

$$
\theta^{t}(z)=1+\sum_{1}^{\infty} R_{t}(m) e(m z) .
$$

The theta-function has the property that it responds to identities under elements of $G_{3}$, namely

$$
\begin{aligned}
& \theta(z+2)=\theta(z), \\
& \theta(-1 / z)=\theta(z)(z / i)^{1 / 2} .
\end{aligned}
$$

In order to accommodate the whole modular group $G$ we must introduce

$$
\theta(c, d ; z)=\sum_{n=-\infty}^{\infty} e\left[z(n+c / 2)^{2}+n d\right]
$$

where $c$ and $d$ are integers. Then

$$
\begin{aligned}
& \theta(c, d ; z+1)=\theta(c, c+d+1 ; z) e\left(c^{2} / 4\right), \\
& \theta(c, d ;-1 / z)=\theta(d, c ; z) e(-c d / 2)(z / i)^{1 / 2} .
\end{aligned}
$$

Since

$$
\theta(c, d+2 ; z)=(-)^{d} \theta(c+2, d ; z)=\theta(c, d ; z)
$$

it is clear that at most, four theta functions are involved. Moreover,

$$
\theta(1,1 ; z) \equiv 0
$$

by cancellation of pairs of terms, thus only three are left

$$
\theta(0,0 ; z), \quad \theta(0,1 ; z), \quad \theta(1,0 ; z)
$$

and we identify

$$
\theta(0,0 ; z)=\theta(z) .
$$


We speak of a three valued modular function $f(c, d ; z)$ "transforming like $\theta$ " if it has the same functional equation. We speak of such a $f(c, d, z)$ as "single-valued under $G$ " if its three values are the same regardless of $c$ and $d$ (excl. $c=1, d=1$ ).

Finally by writing the first few terms, we find, that as $z \rightarrow i \infty$,

$$
\begin{aligned}
& \theta(0, d ; z) \rightarrow 1 \quad(d=0 \text { or } 1), \\
& \theta(1,0 ; z) \rightarrow 0 .
\end{aligned}
$$

6. The singular series. We henceforth assume that $t \equiv 0(\bmod 4)$. Since eighth roots of unity enter into (5.6) and (5.7) we have the general situation

$$
\theta^{t}(c, d ; z)=\theta^{t}\left(c_{g}, d_{g} ; g(z)\right)(r z+s)^{-t / 2} \chi^{t / 2}
$$

where

$$
g(z)=(p z+q) /(r z+s)(\in G)
$$

and $c_{g}, d_{g}$ are another pair of integers $(\bmod 2)$ while $\chi= \pm 1$ depending on $g(z)$ and $c$ and $d$. In particular we can show

$$
\theta^{t}(0,0 ; z)=\theta^{t}\left(c_{g}, d_{g} ; g(z)\right)(r z+s)^{-t / 2} \chi(g)^{t / 4}
$$

where

$$
c_{g}=0 \text { precisely when } r z+s \equiv 1 \text { or } z(\bmod 2)
$$

and in that case

(6.5) $\chi(g)=1$ or -1 according as $r z+s \equiv 1$ or $z(\bmod 2)$.

Hence if $z \rightarrow-s / r$, an arbitrary rational fraction, then

$$
\theta^{t}(0,0 ; z) \approx \begin{cases}(r z+s)^{-t / 2} & \text { if } \quad(r, s) \equiv(0,1)(\bmod 2) \\ (-)^{t / 4}(r z+s)^{-t / 2} & \text { if } \quad(r, s) \equiv(1,0)(\bmod 2) \\ 0 & \text { if } \quad(r, s) \equiv(1,1)(\bmod 2)\end{cases}
$$

(Here " $\approx 0$ " means "small zero" of $(r z+s)^{-t / 2}$.) Hence a "singular series" not unlike the Eisenstein series is suggested

$$
S_{t}(0,0 ; z)=\sum_{r / s}(r z+s)^{-t / 2}(-1)^{r t / 4} \text { ( } r s \text { even). }
$$

Clearly, ignoring sign, $\pm S_{\iota}(0,0, z)$ will have three values under $G$ (although invariant under $G_{3}$ ),

$$
S_{t}(0,0 ; z), \quad S_{t}(0,1 ; z), \quad S_{t}(1,0 ; z) .
$$

We can evaluate $S_{t}(0,0 ; z)$ by Fourier series analogously with the 
Eisenstein series. We obtain

$$
S_{t}(0,0 ; z)=1+\sum_{1}^{\infty} S_{t}(m) e(m z)
$$

where $S_{t}(m)$ is the "singular approximation" to $R_{t}(m)$. It has the form

(6.10) $S_{t}(m)=D_{t}\left[\right.$ odd quot. $\sigma_{t / 2-1}(m)+(-)^{t / 4}$ exc. $\left.\sigma_{t / 2-1}(m / 2)\right]$,

where

$$
\begin{aligned}
D_{t} & =\pi^{t / 2} /\left[\Gamma(t / 2)\left(1-2^{-t / 2}\right) \zeta(t / 2)\right] \\
& =8,16,8, \cdots, \text { for } t=4,8,12, \cdots,
\end{aligned}
$$

and two divisor functions arise. First the "odd-quotient" type occurs (6.12) odd quot. $\sigma_{t / 2-1}(m)=\sum n^{t / 2-1}$ (over $n$ for which $m / n$ is odd); and then the "excess-of-even-divisors-over-odd" type occurs

$$
\text { exc. } \left.\sigma_{t / 2-1}(m)=\sum n^{t / 2-1}(-1)^{n} \text { (over } n \mid m\right) \text {. }
$$

We follow the convention that for a number-theoretic function $\sigma(\xi)=0$ if $\xi$ fails to be integral, so that (6.10) is a different formula for $m$ even than for $m$ odd.

When $t \equiv 0(\bmod 8)$, we can form a complete Eisenstein series

$$
\left.S_{t}^{0}(z)=\frac{1}{2} \sum(r z+s)^{-t / 2} \quad \text { (over all }(r, s)=1\right) .
$$

Here, $S_{t}^{0}(z)$ has only one value (not three) under $G$, indeed it differs by the constant factor $2 \zeta(t / 2)$ from $E_{t / 2}(z)$ as defined in (4.4). Then; we expand and find

$$
S_{t}^{0}(z)=1+\sum_{1} S_{t}^{0}((m)) e(2 m z)
$$

where

$$
\begin{aligned}
S_{t}^{0}((m)) & =\left\{(2 \pi)^{t / 2} /[\Gamma(t / 2) \zeta(t / 2)]\right\} \sigma_{t / 2-1}(m) \\
& =240 \sigma_{3}(m) \quad \text { when } \quad t=8 .
\end{aligned}
$$

The fact that this construction requires $t \equiv 0(\bmod 8)$ is quite significant in extension to several variables, where the "more restricted" condition $t \equiv 0(\bmod 4)$ seems as satisfactory (see $\$ 11$ below).

7. Validity of singular series. Clearly $R_{t}(1)=2 t$ since

$$
1=\cdots 0+\cdots+( \pm 1)^{2}+\cdots 0+\cdots
$$


Hence the singular approximation is valid only if $m=4$ or 8 . Indeed,

$$
\begin{array}{lll}
R_{4}(m)=S_{4}(m) & \text { or } \quad \theta^{4}(0,0 ; z)=S_{4}(0,0 ; z), \\
R_{8}(m)=S_{8}(m) & \text { or } \quad \theta^{8}(0,0 ; z)=S_{8}(0,0 ; z) .
\end{array}
$$

Actually a "bonus" occurs when $t=8$, because

$$
S_{8}^{0}((m))=\text { No. of Rep. of } 2 m=\phi_{0}\left(x_{1}, \cdots, x_{8}\right)
$$

where

$$
\begin{aligned}
\phi_{0}\left(x_{1}, \cdots, x_{8}\right)= & 2 \sum_{1}^{8} x_{j}^{2}+2 x_{6}\left(x_{1}+x_{2}+x_{3}\right)+2 x_{6}\left(x_{1}-x_{2}+x_{4}\right) \\
& +2 x_{7} x_{3}+2 x_{8}\left(x_{1}-x_{3}-x_{4}\right) .
\end{aligned}
$$

This form is less mysterious if we write it as

$$
\begin{aligned}
2 \phi_{0} & =y_{1}^{2}+\cdots+y_{8}^{2}, \\
y_{1}+y_{5} & \equiv y_{2}+y_{6} \equiv y_{3}+y_{7} \equiv y_{4}+y_{8} \\
& \equiv y_{6}+y_{6}+y_{7}+y_{8}(\bmod 2) .
\end{aligned}
$$

It is clear from this that $\phi_{0}$ has determinant 1 , just like $\sum_{1}^{8} x_{j}^{2}$, but $\phi_{0}$ represents only even numbers. Actually

$$
\begin{aligned}
S_{8}^{0}(z) & =\sum e\left(z \phi_{0}\left(x_{1}, \cdots, x_{8}\right)\right) \text { (summed over integral 8-tuples) } \\
& =\sum_{1}^{\infty} e(2 m z) S_{8}^{0}((m)) .
\end{aligned}
$$

8. Properties of the cusp form. For $t=12, R_{12}(m) \neq S_{12}(m)$, but as Glaisher discovered, one can write

$$
\theta^{12}(z)=S_{12}(z)+16 \Delta^{1 / 2}(z)
$$

where

$$
\Delta(z)=e(2 z) \prod_{j=1}^{\infty}(1-e(2 j z))^{24},
$$

the generating function of the Ramanujan tau-series. It can be verified that

$$
\Delta^{1 / 2}(z)=e(z)-12 e(3 z)-\cdots=\sum_{1}^{\infty} \Lambda(m) e(m z)
$$

has coefficients which are multiplicative and (trivially) which vanish 
for $m$ even. Thus,

$$
R_{12}(m)=S_{12}(m) \text { for even } m(>0) .
$$

The quantity $\Delta^{1 / 2}(z)$ is called a "cusp-form" since it vanishes at $z=$ rational (the "cusps" of $\bar{D}_{\boldsymbol{z}}$ at $z=1$ and $\infty$ in particular). It must vanish there since the singular series removes the "main part" of $\theta^{12}(z)$.

The most important property of $\Delta^{1 / 2}(z)$ for us is the following:

$$
-64 \theta^{12}(z) / \Delta^{1 / 2}(z)=\psi(z),
$$

where $\psi(z)$ is the mapping function alluded to in $\S 4$ above. Thus the cusp forms are useful for their conformal mapping properties. To obtain $\psi(z)$ from $\theta^{8}(z)$ would be more difficult:

$$
\psi(z)=\frac{1}{2} \theta(0,0 ; z)^{8} /\left\{\theta(0,0 ; z)^{8}-\theta(0,1 ; z)^{8}-\theta(1,0 ; z)^{8}\right\} .
$$

Thus the cusp forms might be "number-theoretically" unwelcome but they are "function-theoretically" very useful. This holds for modular functions of several variables just as well.

\section{The Quadratic modular Functions}

9. The Hilbert domain. The modular subdivision was extended to several variables by Hilbert and Blumenthal around 1900 and suitable modular functions were defined by Hecke and Siegel from 19101940 approximately. This process was carried out with very little knowledge of the actual shape of the fundamental domain.

Let us narrow our considerations to a real quadratic field $Q(\sqrt{ } k)$ ( $k$ square-free $>1$ ) with fundamental unit $\epsilon_{0}(>1)$ and fundamental totally positive unit $\epsilon_{+}(>1)$. The integers are denoted by $\mathfrak{D}$. (An integer $\mu$ of $Q(\sqrt{ } k)$ is called totally positive, and written $\mu \gg 0$, when $\mu>0$ and the conjugate $\mu^{\prime}>0$.) Thus when

$$
\begin{array}{ll}
k=2, & \epsilon_{0}=1+\sqrt{ } 2, \quad \epsilon_{+}=2 \\
k=3, & \epsilon_{0}=2+\sqrt{ } 3,
\end{array}
$$

We consider $\mathfrak{U}$ the cartesian product of two independent half-planes

$$
\mathfrak{u}: \operatorname{Im} \tau>0, \quad-\operatorname{Im} \tau^{\prime}>0 .
$$

We define the (complete) Hilbert modular group as the transformations $\mathfrak{g}(\tau)$ of $\mathfrak{u}$

$$
\text { (5): } \tau \rightarrow \mathfrak{g}(\tau), \quad \tau^{\prime} \rightarrow \mathfrak{g}^{\prime}\left(\tau^{\prime}\right)
$$

where 


$$
\mathfrak{g}(\tau)=\frac{\alpha \tau+\beta}{\gamma \tau+\delta}, \quad g^{\prime}\left(\tau^{\prime}\right)=\frac{\alpha^{\prime} \tau^{\prime}+\beta^{\prime}}{\gamma^{\prime} \tau^{\prime}+\delta^{\prime}}
$$

and $\alpha, \beta, \gamma, \delta$ (with the conjugates $\left.\alpha^{\prime}, \beta^{\prime}, \gamma^{\prime}, \delta^{\prime}\right)$ are integers of $Q(\sqrt{ } k)$ satisfying

$$
\alpha \delta-\beta \gamma=\epsilon, \quad \alpha^{\prime} \delta^{\prime}-\beta^{\prime} \gamma^{\prime}=\epsilon^{\prime}
$$

with $\epsilon, \epsilon^{\prime}$ conjugate totally positive units. Clearly, it will suffice to write (9.3), (9.4), (9.5) as equations on $\tau$ alone.

A more immediately applicable group (for theta-functions) is the restricted Hilbert group.

$$
\text { (S: } \tau \rightarrow \mathfrak{g}(\tau), \quad \mathfrak{g}(\tau)=(\alpha \tau+\beta) /(\gamma \tau+\delta)
$$

where, this time,

$$
\alpha \delta-\beta \gamma=\epsilon^{2} \quad \text { (є a unit). }
$$

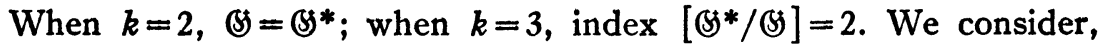

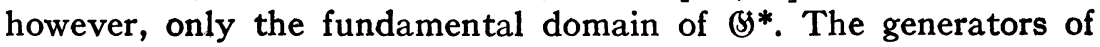
(5) are $\tau \rightarrow \tau+\nu(\nu \in \mathcal{D}), \tau \rightarrow-1 / \tau$ and $\tau \rightarrow \epsilon_{+} \tau$ (or $\tau \rightarrow \epsilon_{0}^{2} \tau$ for (S) analogously with (2.4), if we assume the euclidean algorithm.

We assume $Q(\sqrt{ } k)$ has class number 1 . Then the fundamental domain can be defined as before by generalizing $G_{\infty}, D_{\infty}$, and $D_{0}$. Thus

$$
\mathbb{S}_{\infty}^{*}: \tau \rightarrow \epsilon \tau+\nu, \quad \epsilon \gg 0
$$

and $\mathfrak{D}_{\infty}^{*}$, a fundamental domain under $\mathcal{E S}_{\infty}^{*}$ is

$$
\mathscr{D}_{\infty}^{*}:\left\{\begin{array}{l}
\epsilon_{+}^{-1}<\operatorname{Im} \tau /-\operatorname{Im} \tau^{\prime} \leqq \epsilon_{+}, \quad\left(\tau, \tau^{\prime}\right) \in \mathfrak{U} . \\
\left(\operatorname{Re} \tau, \operatorname{Re} \tau^{\prime}\right) \in \mathfrak{P}(\sqrt{ } k)
\end{array}\right.
$$

where $\mathfrak{B}(\sqrt{ } k)$ is a plane parallelogram (or other fundamental domain) for the translation group $\left(\operatorname{Re} \tau, \operatorname{Re} \tau^{\prime}\right) \rightarrow\left(\operatorname{Re} \tau+\nu, \operatorname{Re} \tau^{\prime}+\nu^{\prime}\right)$ for integer $v$ in $Q(\sqrt{ } k)$. Likewise, we define the norm symbol formally as

$$
N(\gamma \tau+\delta)=(\gamma \tau+\delta)\left(\gamma^{\prime} \tau^{\prime}+\delta^{\prime}\right)
$$

so that the definition can be made as follows:

(9.11) $\mathfrak{D}_{0}^{*}:|N(\gamma \tau+\delta)|^{2} \geqq 1$ for all $(\gamma, \delta)=1, \quad\left(\tau, \tau^{\prime} \in \mathfrak{U}\right)$.

The fundamental domain of $\mathbb{S}^{*}$ can be taken as

$$
\mathfrak{D}^{*}=\mathfrak{D}_{0}^{*} \cap \mathfrak{D}_{\infty}^{*}
$$


The fact that the class number is 1 is reflected in the fact that the boundary of $\mathfrak{D}_{0}^{*}$, the "floor," lies above the real hyperplane (Im $\tau$ $\left.=-\operatorname{Im} \tau^{\prime}=0\right)$. Indeed, it can be shown [1], [6] that

$$
(\operatorname{Im} \tau)\left(-\operatorname{Im} \tau^{\prime}\right)>2 / d
$$

for $d$ (the discriminant) $=k$ if $k \equiv 1$ and $4 k$ if $k \neq \equiv 1(\bmod 4)$.

According to a computation made by the author [6], the floor $\partial \mathfrak{D}_{0}^{*} \cap \mathfrak{D}_{\infty}^{*}$ cannot consist of one piece, however the fundamental domain may be chosen, except for $k=5$ (where Götzky [9] showed the floor can be made to consist of the piece $|N(\tau)|^{2}$ i.e., $\left|\tau \tau^{\prime}\right|^{2}=1$ ). When $k=2$ or 3 there are at least two pieces, say

$$
\begin{aligned}
& |N(\tau)|^{2}=1 \text {, } \\
& |N(\sqrt{ } 2 \tau+1)|^{2}=1 \text {, and, } \\
& |N(\tau)|^{2}=1, \\
& |N((1+\sqrt{ } 3) \tau+1)|^{2}=1 \text {. }
\end{aligned}
$$

When $k=14$ (no euclidean algorithm) there are at least 7 such pieces. Thus we must be prepared to expect some fairly complicated topological structures in 4-space. (This is the subject of a computation presently under way on the Argonne CDC 3600 computer.)

As a matter of notation we shall write $\mathfrak{D}^{*}(\sqrt{ } k), \mathbb{S}(\sqrt{ } k)$, etc., instead of $\mathfrak{D}^{*}$, $\$$, etc., when the context is not clear.

The problems of compactification ( $\$ 3$ ) and of algebraic manifolds (\$4) are now of a higher order of magnitude than the numbertheoretic identities. We therefore defer them.

10. Representations as sums of squares. We consider the problem of representing an integer $\mu$ of $Q(\sqrt{ } k)$ as the sum of squares of $t$ integers $\nu_{1}, \cdots, \nu_{t}$ in $Q(\sqrt{ } k)$. Let $R_{t}(\sqrt{ } k, \mu)$ be the number of such representations. We let $\mathfrak{D}^{+}$denote the totally positive subset of $\mathfrak{D}$. Clearly $\mu \in \mathfrak{D}^{+}$if we are to have a reasonable problem.

To further specify matters, let us assume

$$
k \not \equiv 1 \text {, i.e., } k \equiv 2 \text { or } 3(\bmod 4) \text {. }
$$

(Here we note that the case $k=5$ leads to 10 theta-functions and presents a rather poor analogy to the rational case, although that case serves as a model for what we do here.) This condition (10.1) leads to the ideal factorization of 2 as a square of a principal factor,

$$
2=2_{1}^{2} \text {. }
$$

We take $\omega=k+\sqrt{ } k-2$ so that

$$
\omega=\sqrt{ } 2 \text { when } k=2 ;
$$$$
\omega=1+\sqrt{ } 3 \text { when } k=3 .
$$

Thus $(\omega)=21$ in these cases. 
Also it is not possible to consider $R_{\imath}(\sqrt{ } k, \mu)$ unless $\mu=A+2 B \sqrt{ } k$, i.e.,

$$
\mu \in \mathfrak{D}_{2}^{+}
$$

where $\mathfrak{D}_{2}$ is the subset of $\mathfrak{D}$ where $\mu \equiv 0$ or $1(\bmod 2)$ and $\mathfrak{D}_{2}^{+}$is the totally positive subset of $\mathfrak{D}_{2}$. (Thus $A^{2}-4 B^{2} k=N(\mu)>0$.)

We introduce the formal symbol

$$
e(\lambda \tau)=\exp \pi i\left(\lambda \tau-\lambda^{\prime} \tau^{\prime}\right) / 2 \sqrt{ } k
$$

where $\lambda \in Q(\sqrt{ } k)$ and $\tau \in \mathfrak{U}$. Here, if $\lambda \in \mathcal{D}$, then

$$
e(\lambda)=1 \text { exactly when } \lambda \in \mathfrak{D}_{2},
$$

(10.7) $e(\lambda \tau) \rightarrow 0$ as $\tau \rightarrow i \infty,\left(\tau^{\prime} \rightarrow-i \infty\right)$ exactly when $\lambda \in D^{+}$.

These conditions are analogous to those of $e(n z)$, (as in (3.3)). We have the additional symmetry property $e(\lambda \tau)=e\left(-\lambda^{\prime} \tau^{\prime}\right)$.

If we define, for some $k$,

$$
\theta(\tau)=\sum e\left(\nu^{2} \tau\right)(\text { over } \nu \in D),
$$

then

$$
\theta(\tau)=1+\sum R_{t}(\sqrt{ } k, \mu) e(\mu \tau) \quad\left(\text { over } \mu \in \mathfrak{D}_{2}^{+}\right) .
$$

When we show $R_{t}(\sqrt{ } k, \mu)>0$ then all "congruentially eligible" integers actually are expressible as the sum of $t$ squares (as we do for $t=4, k=2$ ).

We formulate

$$
\theta(c, d ; \tau)=\sum e\left[\tau(\nu+c \omega / 2)^{2}+d \nu \omega\right] \quad(\text { over } \nu \in \mathfrak{D})
$$

so that $\theta(c, d ; \cdots)$ is determined by $c$ and $d(\bmod 2)$ and, of course,

$$
\theta(0,0 ; \tau)=\theta(\tau) \text {. }
$$

Then four theta-functions (not three!) are actually involved:

$$
\begin{aligned}
\theta(c, d ; \tau+1) & =\theta(c, d+c ; \tau) e_{1}, \\
\theta(c, d ; \tau+\omega) & =\theta(c, d+1 ; \tau) e_{2}, \quad(\omega=k+\sqrt{ } k-2), \\
\theta\left(c, d ; \epsilon^{2} \tau\right) & =\theta(c, d ; \tau) e_{3}, \\
\theta(c, d ;-1 / \tau) & =\theta(d, c ; \tau) N(\tau)^{1 / 2} e_{4}
\end{aligned}
$$

where $e_{1}, e_{2}, e_{3}, e_{4}$ are fourth roots of unity rather than eighth roots as in the rational case (see [2]). Hence if 


$$
t \equiv 0(\bmod 4)
$$

the signs of $\theta^{t}$ present no problem. Also, as $\tau \rightarrow i \infty\left(\tau^{\prime} \rightarrow-i \infty\right)$,

$$
\begin{aligned}
& \theta(c, d ; \tau) \rightarrow 1 \quad \text { if } \quad c=0, \\
& \theta(c, d ; \tau) \rightarrow 0 \quad \text { if } \quad c=1,
\end{aligned}
$$

by actual examination of leading terms.

11. The singular series. We now make the further restriction on $k$ that the euclidean algorithm for $Q(\sqrt{ } k)$ is valid (so that the class number is definitely 1$)$. Then the operations in (10.11) are seen to generate $\mathbb{S}(\sqrt{ } k)$ the restricted Hilbert group (9.7) by analogy with (2.4). We also assume

$$
\epsilon_{0} \not \equiv 1(\bmod 2)
$$

which is true for $k=2,3,7$ (but false for any other even $k>2$ where $Q(\sqrt{ } k)$ has class number 1$)$. From the property (11.1) if $\gamma$ is odd $\left(N(\gamma)\right.$ odd) then $\gamma$ or $\epsilon_{0} \gamma$ belongs to $\mathfrak{D}_{2}$.

We saw that $\theta^{t}$ presents no sign problem. Thus if $\mathfrak{g}(\tau) \in(5 *$

$$
\mathfrak{g}(\tau)=(\alpha \tau+\beta) /(\gamma \tau+\delta)
$$

then as in (6.3), with $t \equiv 0(\bmod 4)$

$$
\theta^{t}(0,0 ; \tau)=\theta^{t}\left(c_{\mathfrak{g}}, d_{\mathfrak{g}} ; \mathfrak{g}(\tau)\right) N(\gamma \tau+\delta)^{-t / 2} .
$$

It can be shown that the substitutions $\mathfrak{g}(\tau)$ for which $c_{\mathfrak{g}}=0$ are those for which $\gamma$ and $\delta \in \mathfrak{D}_{2}$. (Recall $(\gamma, \delta)=1$ so at least one of these is odd.) Thus, we obtain the singular series analogous with (6.7).

(11.4) $S_{t}(\sqrt{ } k, \tau)=\sum N(\gamma \tau+\delta)^{-t / 2}, \gamma$ and $\delta \in \mathfrak{D}_{2}, \quad(\gamma, \delta)=1$.

At the same time we can formulate, analogously with (6.14)

$$
S_{t}^{(0)}(\sqrt{ } k, \tau)=\sum N(\gamma \tau+\delta)^{-t / 2}, \quad(\gamma, \delta)=1 .
$$

It is understood in such summations that we do not repeat associates, i.e., if $\gamma_{1}=\epsilon \gamma, \delta_{1}=\epsilon \delta$ where $\epsilon$ is a unit then we do not repeat both $(\gamma, \delta)$ and $\left(\gamma_{1}, \delta_{1}\right)$. Clearly $S_{\imath}^{(0)}(\sqrt{ } k, \tau)$ has all four values equal under (5* (unlike $\theta^{t}(\tau)$ in (10.11)).

We find, analogously with (6.10) and (6.15)

$$
S_{t}(\sqrt{ } k, \tau)=1+\sum S_{t}(\sqrt{ } k, \mu) e(\mu \tau), \quad \mu \in \mathfrak{D}_{2}^{+}
$$

where

(11.7) $S_{t}(\sqrt{ } k, \mu)=D_{t}(\sqrt{ } k)\left[\right.$ odd quot. $\left.\sigma_{t / 2-1}(\mu)+2^{t / 2-1} \operatorname{exc} \sigma_{t / 2-1}\left(\mu / 2_{1}^{3}\right)\right]$ 
and, as before (restricting sums so that each ideal ( $\nu$ ) occurs once), odd quot. $\sigma_{t / 2-1}(\mu)=\sum_{(\nu)}|N(\nu)|^{t / 2-1}$ where $\mu / \nu(\in D)$ is odd,

$$
\text { exc. } \begin{aligned}
\sigma_{t / 2-1}(\mu) & =\sum_{(\nu)}|N(\nu)|^{t / 2-1}(-1)^{N(\nu)} \text { where } \nu / \mu \text { in } \mathfrak{D} . \\
D_{t}(\sqrt{ } k) & =2 \pi^{t} /\left[(4 k)^{(t-1) / 2} \zeta(\sqrt{ } k, t / 2)\left(1-2^{-t / 2}\right)\right] .
\end{aligned}
$$

Of course (11.7) is "two formulas" depending on whether or not $8 \mid N(\mu)$, and $\zeta(\sqrt{ } k, t / 2)$ is the zeta function for the field. We see $D_{4}(\sqrt{ } k)=8$ for $k=2$ and 4 for $k=3$.

Furthermore

$$
S_{t}^{0}(\sqrt{ } k, \tau)=1+\sum S_{t}^{0}((\sqrt{ } k, \mu)) e(2 \mu \tau) \quad\left(\operatorname{over} \mu \in D^{+}\right)
$$

where

$$
S_{t}^{0}((\sqrt{ } k, \mu))=D_{t}^{0}(\sqrt{ } k) \sigma_{t / 2-1}(\mu),
$$

and

$$
D_{t}^{0}(\sqrt{ } k)=2(2 \pi)^{t} /\left[(4 k)^{(t-1) / 2} \zeta(\sqrt{ } k, t / 2)\right]
$$

so that

$$
D_{4}^{0}(\sqrt{ } k)=48 \text { for } k=2 \text { and } 24 \text { for } k=3 .
$$

The situation is the following for $t=4$ : For $k=2$,

$$
\theta^{4}(\tau)=S_{4}(\tau) \text { and } R_{4}(\sqrt{ } 2, \mu)=S_{4}(\sqrt{ } 2, \mu) .
$$

Thus it is easily seen that $R_{4}(\sqrt{ } 2, \mu)>0$ if $\mu \in \mathfrak{D}_{2}^{+}$so that every integer $A+2 B \sqrt{ } 2$, with $A>|2 B \sqrt{ } 2|$, is the sum of four squares in $\leqq(\sqrt{ } 2)$. For $k=3$, then a cusp form $\Lambda(\tau)$ exists for which

$$
\theta^{4}(\tau)=S_{4}(\tau)+4 \Lambda(\tau)
$$

and an error-function $L(\mu)$ exists $\left(\mu \in \mathfrak{D}_{2}^{+}\right)$

$$
\begin{gathered}
R_{4}(\sqrt{ } 3, \mu)=S_{4}(\sqrt{ } 3, \mu)+4 L(\mu), \\
\left.\Lambda(\tau)=\sum L(\mu) e(\mu \tau) \quad \text { (over } \mu \in \mathfrak{D}_{2}^{+}\right) .
\end{gathered}
$$

This is not surprising since for $\theta^{t}=S_{t}$ we should need $D_{t}(\sqrt{ } k)=2 t$. This is seen to be impossible unless $k=2, t=4$ because the $\zeta$-function is close to 1 anyway and $D_{t}(\sqrt{ } k)$ is noticeably less than $2 t$ otherwise.

The singular series $S_{4}^{0}(\sqrt{ } k, \tau)$ corresponds to the following even forms of determinant 1 (see [15]) 


$$
\begin{aligned}
k=2, \quad \phi_{0}\left(\xi_{1}, \cdots, \xi_{4}\right)= & 2\left(\xi_{1}^{2}+\xi_{1} \xi_{2}+\xi_{2}^{2}+\xi_{3}^{2}+\xi_{3} \xi_{4}+\xi_{4}^{2}\right) \\
& +2 \sqrt{ } 2\left(\xi_{1} \xi_{4}+\xi_{4} \xi_{2}+\xi_{2} \xi_{3}\right),
\end{aligned}
$$

or $2 \phi_{0}=\eta_{1}^{2}+\eta_{2}^{2}+\eta_{3}^{2}+\eta_{4}^{2}$ where

$$
\begin{gathered}
\eta_{1} \equiv \eta_{3}+\sqrt{ } 2 \eta_{4}, \quad \eta_{2} \equiv \eta_{3} \sqrt{ } 2+\eta_{4}(\bmod 2) ; \\
k=3, \quad \phi_{0}\left(\xi_{1}, \cdots, \xi_{4}\right)= \\
2 \xi_{1}^{2}+2 \xi_{1} \xi_{2} \sqrt{ } 3+2 \xi_{2}^{2}+2 \xi_{3}^{2} \\
+2 \xi_{3} \xi_{4} \sqrt{ } 3+2 \xi_{4}^{2}
\end{gathered}
$$

or $2 \phi_{0}=\eta_{1}^{2}+\eta_{2}^{2}+\eta_{3}^{2}+\eta_{4}^{2}$ where

$$
\eta_{1} \equiv \eta_{2} \sqrt{ } 3, \quad \eta_{3} \equiv \eta_{4} \sqrt{ } 3(\bmod 2) .
$$

In each case $S_{4}^{0}((\sqrt{ } k, \mu))$ is the number of representations of

$$
\phi_{0}=2 \mu \text {, }
$$

and

$$
S_{4}^{0}(\sqrt{ } k, \tau)=1+\sum S_{4}^{0}((\sqrt{ } k, \mu)) e(2 \mu \tau) \quad\left(\operatorname{over} \mu \in D^{+}\right) .
$$

The modular function (11.19), like its singular series (11.5), keeps transforming into itself under $\&$ (all four values coincide). It will be needed later on $(\$ 16)$ in an analytic context.

12. The cusp form for $\theta^{4}$ over $Q(\sqrt{ } 3)$. We now consider the cusp form

$$
\Lambda(\tau)=\sum L(\mu) e(\mu \tau) .
$$

The coefficients are multiplicative, somewhat like the Ramanujan and Glaisher functions. Indeed [3], analogously with (8.4)

$$
L(\mu)=0 \text {, i.e., } R_{4}(\sqrt{ } 3, \mu)=S_{4}(\sqrt{ } 3, \mu) \quad\left(\mu \in \mathfrak{D}_{2}^{+}\right)
$$

whenever

$$
2^{3} \mid N(\mu), 3^{\text {odd }}\left\|N(\mu), r^{\text {odd }}\right\| N(\mu) \quad(\text { prime } r \equiv-1 \bmod 12) .
$$

(Here the symbol " $p^{t} \| a$ " means " $\left.p^{t} \mid a, p^{t+1} \nmid a . "\right)$ We can see (12.2) and (8.4) as analogous in that a "bad" prime divisor makes the "exact" formula valid!

The presence of the cusp form $\Lambda(\tau)$ can be explained by the fact that three forms of determinant 1 share the genus of

$$
\phi_{1}(\xi)=\xi_{1}^{2}+\xi_{2}^{2}+\xi_{3}^{2}+\xi_{4}^{2} .
$$

They are, according to M. Kneser (private communication) 


$$
\phi_{2}(\xi)=\xi_{1}^{2}+\xi_{2}^{2}+2 \xi_{3}^{2}+2 \sqrt{ } 3 \xi_{3} \xi_{4}+2 \xi_{4}^{2}
$$

and

$$
\begin{aligned}
\phi_{3}(\xi)= & 3\left(\xi_{1}^{2}+\xi_{2}^{2}+\xi_{3}^{2}+\xi_{4}^{2}\right)+2(1+\sqrt{ } 3)\left(\xi_{1} \xi_{3}-\xi_{2} \xi_{4}\right) \\
& +2(1-\sqrt{ } 3)\left(\xi_{1} \xi_{4}+\xi_{2} \xi_{3}\right) .
\end{aligned}
$$

Actually

$$
2 \phi_{2}=\eta_{1}^{2}+\eta_{2}^{2}+\eta_{3}^{2}+\eta_{4}^{2}
$$

where

$$
\eta_{1} \equiv \eta_{2}, \quad \eta_{3} \equiv \sqrt{ } 3 \eta_{4}(\bmod 2)
$$

and

$$
2 \phi_{3}=\eta_{1}^{2}+\eta_{2}^{2}+\eta_{3}^{2}+\eta_{4}^{2}
$$

where each

$$
\eta_{j}(1+\sqrt{ } 3) \equiv \eta_{1}+\eta_{2}+\eta_{3}+\eta_{4}(\bmod 2) .
$$

It also turns out that

$$
\begin{aligned}
S_{4}(\sqrt{ } 3, \mu) & =\text { No. of Rep. of }\left\{\phi_{2}(\xi)=\mu\right\}, \\
S_{4}(\sqrt{ } 3, \mu)-4 L(\mu) & =\text { No. of Rep. of }\left\{\phi_{3}(\xi)=\mu\right\} .
\end{aligned}
$$

Hence we have a total of three theta functions satisfying the system satisfied by $\theta^{4}(c, d ; \tau)$ together with $S_{4}^{0}(\sqrt{ } 3, \tau)$ (which has all four conjugates equal). Of course if $\mu\left(\in \mathfrak{D}_{2}^{+}\right)$satisfies (12.3) then $\phi_{1}, \phi_{2}, \phi_{3}$ each represent $\mu$ (an equal number) $S_{4}(\sqrt{ } 3, \mu)$ times.

We can also define $\Lambda(c, d ; \tau)$ by the functional equations for $\theta^{4}(c, d ; \tau)$ by starting with $\Lambda(0,0 ; \tau)=\Lambda(\tau)$. We then find

$$
\Lambda\left(c, d ; \epsilon_{+} \tau\right)=\Lambda(c+1, d+1 ; \tau)
$$

so that even under the complete Hilbert group $\mathbb{S}^{*}, \Lambda(\tau)$ is fourvalued. Furthermore,

$$
S_{4}^{0}(\sqrt{ } 3, \tau)=S_{4}^{0}\left(\sqrt{ } 3, \tau \epsilon_{+}\right)
$$

thus the four quantities

$$
\Lambda(c, d ; \tau) / S_{4}^{0}(\sqrt{ } 3, \tau), \quad c, d(\bmod 2)
$$

are permuted by operations of $(5 *$.

Now, reminiscent of the manner in which the resolvent cubic is derived, we set [3] 


$$
\begin{aligned}
\Lambda(0,0 ; \tau)+\Lambda(1,1 ; \tau)-\Lambda(1,0 ; \tau)-\Lambda(0,1 ; \tau) & =P(\tau), \\
& \Lambda(0,0 ; \tau)-\Lambda(1,1 ; \tau)+\Lambda(1,0 ; \tau)-\Lambda(0,1 ; \tau)=M(\tau), \\
& \Lambda(0,0 ; \tau)-\Lambda(1,1 ; \tau)-\Lambda(1,0 ; \tau)+\Lambda(0,1 ; \tau)=K(\tau), \\
\text { while } \quad \Lambda(0,0 ; \tau)+\Lambda(1,1 ; \tau)+\Lambda(1,0 ; \tau)+\Lambda(0,1 ; \tau) & =0 .
\end{aligned}
$$

Thus if we set

$$
4 \Lambda(c, d ; \tau)= \pm \sqrt{ } P^{2}(\tau) \pm \sqrt{ } M^{2}(\tau) \pm \sqrt{ } K^{2}(\tau)
$$

(with four choices of sign having positive product), we find $P^{2}(\tau)$, $M^{2}(\tau), K^{2}(\tau)$ satisfy the "resolvent cubic" of the biquadratic whose four roots are $\Lambda(c, d ; \tau)$. These three quantities have 3 ! permutations.

It can be shown that $P^{2}(\tau), M^{2}(\tau), K^{2}(\tau)$ are each kept invariant under a subgroup $\mathbb{S}_{6}^{*}(\sqrt{ } 3)$ of $\mathbb{S}^{*}(\sqrt{ } 3)$ defined by

$$
\mathfrak{G}_{6}^{*}(\sqrt{ } 3): \mathfrak{g}(\tau) \equiv \tau \bmod (1+\sqrt{ } 3) \quad\left(\mathfrak{g}(\tau) \in \mathfrak{f}^{*}(\sqrt{ } 3)\right) .
$$

The proof is straightforward. It consists of noticing that the generators of (F), $\left(\tau \rightarrow \tau+\mathcal{D}, \tau \rightarrow-1 / \tau, \tau \rightarrow \epsilon_{+} \tau\right)$, are congruent to rational transformations $(\tau \rightarrow \tau+1$ or $-1 / \tau) \bmod (1+\sqrt{ } 3)$ and therefore it suffices to check (12.9) for each of the (six) rational transformations (mod 2) (we discover of course that only the identity $\tau \rightarrow \tau$ fails to permute $\left.P^{2}, M^{2}, K^{2}\right)$.

\section{Proofs AND DESCENT PRoCess}

13. Proofs in the rational case by algebraic functions. To appreciate the "luxury" provided by an algebraic manifold, consider the mode of proof of the rational identities.

For example, take (7.2)

$$
\theta^{8}(0,0 ; z)=S_{8}(0,0 ; z) .
$$

To prove this statement, we write

$$
F(z)=\frac{\left[\theta^{8}(0,0 ; z)-S_{8}(0,0 ; z)\right]\left[\theta^{8}(0,1 ; z)-S_{8}(0,1 ; z)\right]}{\left[\left[\theta^{8}(1,0 ; z)-S_{8}(1,0 ; z)\right]\right.} .
$$

By applying the generating substitutions, $(z \rightarrow z+1, z \rightarrow-1 / z)$, we can verify that $F(z)$ is rational on $\bar{D}$ (the compactified fundamental domain); and therefore $F(z)$ is rational in $J(z)$. (We should like to show $F(z)=0$ of course.)

First, note that $F(z)$ might acquire some finite singularities at $z=i$ and $z=\rho$ via the $\left[J^{\prime}(z)\right]^{6}$ factor. By (3.1), however, we see 


$$
F_{0}(z)=J(z)^{4}(J(z)-1)^{3} F(z)
$$

has no finite singularities on the $z$-plane, only possibly $z=i \infty \quad(J=\infty)$. We now verify, from (3.1) again, that

$$
\begin{aligned}
J^{4}(J-1)^{3} / J^{\prime 6} \approx J & =O(e(-2 z)), \\
\theta^{8}(1,0 ; z)-S_{8}(1,0 ; z) & =O(e(z)),
\end{aligned}
$$

since both vanish at $z=i \infty$, and

$$
\theta^{8}(0,1 ; z)-S_{8}(0,1 ; z)=O(1)
$$

since both are finite. Now, by actually comparing the first few terms of $\theta^{8}(0,0 ; z)$ and $S_{8}(0,0 ; z)$ we can check agreement

$$
\theta^{8}(0,0 ; z)-S_{8}(0,0 ; z)=0 \cdot 1+0 \cdot e[z]+0 \cdot e[2 z]+(?)+\cdots
$$

Hence $F_{0}(z) \rightarrow 0$ as $z \rightarrow i \infty$ or $F_{0}(z)$ has no singularities on the compact manifold $\bar{D}$. Clearly $F_{0}(z)$, hence $F(z)$, vanishes identically.

The generalization of this technique to two complex variables is not successful, since the "bicomplex space" is not treated as a manifold.

14. Proofs in the quadratic cases by descent. To prove the quadratic number-theoretic identities (of $\S 11$, etc.) is a more challenging problem. The general genus theory of Siegel [17] provides a uniform method of approach embracing both rational and algebraic fields, but it is not elementary, generally, although work of Pall [4] has shown how to do this when $t=4$, by a reduction to the rational case.

What we do is first establish that the $\theta(c, d ; \tau)$ functions have as "simple zeros" the manifolds [1], on which (just) $\theta(1,1 ; \tau)$ vanishes:

$$
\begin{aligned}
\tau^{\prime}=-\tau & \text { for } k \text { odd and } \epsilon_{0} \not \equiv 1(\bmod 2), \\
\epsilon_{0} \tau=\epsilon_{0}^{\prime} \tau^{\prime} & \text { for } k=2\left(\epsilon_{0}=1+\sqrt{ } 2\right) .
\end{aligned}
$$

Thus it can be shown by a generalization of the Siegel-Götzky method [9], that it suffices to prove an identity by showing it holds on the zero manifolds (14.1) up to a finite number of derivatives with respect to the distance from the zero manifold. The details are quite laborious, but the following "zeroth approximation" will suffice to demonstrate the formalism:

Let $\psi(c, d ; \tau)$ be a quadruple satisfying the same functional equations as $\theta(c, d ; \tau)^{t}$ (like (10.11)) for $k=2$ or 3 . Write

$$
\begin{aligned}
\psi(1,1 ; U,-U) & =\psi(\sqrt{ } 3 ; U) & & \text { for } k=3, \\
\psi\left(1,1 ; \epsilon_{0} U, \epsilon_{0}^{\prime} U\right) & =\psi(\sqrt{ } 2 ; U) & & \text { for } k=2 .
\end{aligned}
$$


Then the functional equations lead to

$$
\begin{aligned}
\psi(\sqrt{ } 3 ; U+\sqrt{ } 3) & =\psi(\sqrt{ } 3 ; U), \\
\psi(\sqrt{ } 3 ;-1 / U) & =\psi(\sqrt{ } 3 ; U) U^{t} ; \\
\psi(\sqrt{ } 2 ; U+1) & =\psi(\sqrt{ } 2 ; U), \\
\psi(\sqrt{ } 2 ;-1 / U) & =\psi(\sqrt{ } 2 ; U) U^{t} .
\end{aligned}
$$

Thus $\psi(\sqrt{ } 3 ; U)$ and $\psi(\sqrt{ } 2 ; U)$ belong to the Hecke or Klein modular group and are now subject to a well-known theory in one variable (see [1], [3]).

Here it is interesting to note that the coefficients of $\psi(1,1 ; U,-U)$ necessarily become sums of coefficients of $\psi\left(1,1 ; \tau, \tau^{\prime}\right)$. Hence they are readily reducible to rational terms. A typical result [5] of the descent process is obtained if $\psi(1,1 ; \tau)$ is taken as $S_{4}^{0}(\sqrt{ } 3, \tau)$ in $\S 11$. Then we find, for example, the number of representations $R(a)$ of

$$
\begin{aligned}
a= & x_{1}^{2}+x_{1} x_{2}+x_{2}^{2}+x_{3}^{2}+x_{3} x_{4}+x_{4}^{2}+x_{6}^{2}+x_{5} x_{6}+x_{6}^{2}+x_{7}^{2} \\
& +x_{7} x_{8}+x_{8}^{2}
\end{aligned}
$$

is the coefficient in $\psi(1,1 ; U,-U)$ of $\exp 2 \pi i a / \sqrt{ } 3$ or

$$
R(a)=24\left[\sigma_{3}(a)+9 \sigma_{3}(a / 3)\right] .
$$

What we are observing in effect is that problems in four squares in a quadratic field project into problems in eight squares in the rational field. Hence it is not surprising that the functional equations (10.11) have only fourth roots of unity in the quadratic case (as compared with eighth roots of unity in the rational case).

By this same token, the sum of two squares might even display remarkable simplification in the case of biquadratic modular forms but no results exist which would substantiate this idea.

\section{REMARKS ON COMPACTIFICATION}

15. Point at infinity and fixed points. The point at $\infty(\tau \rightarrow i \infty$, $\left.\tau^{\prime} \rightarrow-i \infty\right)$ can be compactified trivially as an ideal point but it would not obviously acquire an analytic structure.

We should not take for granted that there is a valid analogue of the rational case, because in the quadratic case, the neighborhood of inside $\mathfrak{D}_{\infty}^{*}$ (see (9.9)) is a "wedge" which might be only a portion on an analytic (bicomplex) neighborhood. Indeed this is so (and the purpose of general procedures or compactification of Baily, Satake, etc., is to generalize to more complicated modular groups than those present here). 
Let us first anticipate that if the neighborhood of $\infty$ in $\mathfrak{D}_{\infty}^{*}$ is not a "full neighborhood," conceivably a function $G$ of $\tau$ and $\tau^{\prime}$ defined in $\mathfrak{D}^{*}$ might have an isolated singularity in $\mathfrak{D}_{\infty}^{*}$ at $\tau=i \infty, \tau^{\prime}=-i \infty$. Actually this will not happen. Let $G\left(\tau, \tau^{\prime}\right)$ be expanded into a convergent power series,

$$
G\left(\tau, \tau^{\prime}\right)=\sum A(\nu) e(\nu \tau) \quad(\nu \in D) .
$$

Then only $\nu \in D^{+}$can occur. To see this, note that (by the group properties under (\$) $A(\nu)=A\left(\nu \epsilon_{+}\right)=\cdots$. Thus if (say) $\nu>0$ and $\nu^{\prime}<0$, then $\nu^{\prime} \epsilon_{+}^{m} \rightarrow-\infty$ and $\lim e\left(\nu \epsilon_{+}^{-m} \tau\right)=\infty$ (as $\left.m \rightarrow \infty\right)$, so that the series (15.1) would never converge for any $\tau$ at all. Now once we know $\nu \in \mathfrak{D}^{+}$(only) then the series (15.1) will converge "all the way to $\infty$ " from any value.

It can be further shown (see Gundlach [10]) that exactly when a group \& has a finite fixed-point then the corresponding domain $D$ does not have an analytic structure at finite points. Actually the case is unlike the rational case where $J^{1 / 3}$ or $(J-1)^{1 / 2}$ serves as a uniformizing parameter. It is more like the impossibility of parametrizing $w=\sqrt{ }\left(z_{1} z_{2}\right)$. Actually Gundlach shows that for $k=3, \mathbb{S}_{6}^{*}$ (as defined in (12.9)) has no fixed point. Its fundamental domain in $\mathfrak{u}$, $\mathfrak{D}_{\mathrm{B}}^{*}(\sqrt{ } 3)$, is six replicas of $\mathfrak{D}^{*}(\sqrt{ } 3)$. We shall compactify just this bicomplex manifold.

16. Introduction of parameters. From $\$ 12$, the following two functions are invariants under $\mathscr{S}_{6}^{*}$,

$$
J_{1}\left(\tau, \tau^{\prime}\right)=\frac{M^{2}(\tau)-K^{2}(\tau)}{\left[S_{4}^{0}(\sqrt{ } 3, \tau)\right]^{2}}, \quad J_{2}\left(\tau, \tau^{\prime}\right)=\frac{P^{2}(\tau)-M^{2}(\tau)}{\left[S_{4}^{0}(\sqrt{ } 3, \tau)\right]^{2}} .
$$

If we expand them, we find the leading terms [3],

$$
\begin{aligned}
& J_{1}\left(\tau, \tau^{\prime}\right)=-4 \zeta_{1}+8 \zeta_{2}+\cdots, \\
& J_{2}\left(\tau, \tau^{\prime}\right)=4 \zeta_{1}+8 \zeta_{2}+\cdots
\end{aligned}
$$

where (recalling $\epsilon_{+}=2+\sqrt{ } 3=(3+\sqrt{ } 3) /(3-\sqrt{ } 3)$ ),

$$
\begin{aligned}
\zeta_{1} & =e[2 \tau]+e\left[2 \epsilon_{+} \tau\right]+e\left[2 \epsilon_{+}^{-1} \tau\right]+\cdots \\
& =\sum_{-\infty}^{\infty} e\left[2 \epsilon_{+}^{n} \tau\right], \\
\zeta_{2} & =e[(3+\sqrt{ } 3) \tau]+e[(3-\sqrt{ } 3) \tau]+\cdots \\
& =\sum_{-\infty}^{\infty} e\left[(3+\sqrt{ } 3) \epsilon_{+}^{n} \tau\right] .
\end{aligned}
$$


In order to use the "..." in (16.2) we must establish orders of magnitude. Actually the orders of magnitude are established by the shape of the wedge in $\mathfrak{D}_{\infty}^{*}$. For example, from $\epsilon_{+}^{-1}<\operatorname{Im} \tau /-\operatorname{Im} \tau^{\prime} \leqq \epsilon_{+}$ (see (9.9) above), it follows that

$$
1 /|e(\tau)| \geqq|e(\sqrt{ } 3 \tau)| \geqq|e(\tau)|
$$

or $1 / e[(|A|+|B|) \tau] \geqq e[(A+B \sqrt{ } 3) \tau] \geqq e[(|A|+|B|) \tau]$. It is clear that $\zeta_{1}$ and $\zeta_{2}$ are unchanged by $\tau \rightarrow \tau+(1+\sqrt{ } 3) \nu$ (the generating translations of $\left.\mathfrak{G}_{6}^{*}\right)$. Furthermore, $\mathfrak{D}_{6}^{*}$ has only two replicas at $\tau=\infty$ (just as $D_{3}$ has two in Figure 1 ) and we can easily verify the following:

Every $\left(\tau, \tau^{\prime}\right)$ in $\mathfrak{D}_{6}^{*}$ determines a unique pair $\left(\zeta_{1}, \zeta_{2}\right)$ for $\operatorname{Im} \tau$ and $-\operatorname{Im} \tau^{\prime}$ large enough. Conversely, however, not every $\left(\zeta_{1}, \zeta_{2}\right)$ is determined by a $\left(\tau, \tau^{\prime}\right)$ in $\mathfrak{D}_{8}^{*}$ (for instance $\left|\zeta_{2}\right| \leqq\left|\zeta_{1}\right|^{2}$ by (16.3), (16.4)). Nevertheless, for every $\zeta_{1}, \zeta_{2}$ both small enough, exactly two pairs

$$
\left(\tau, \tau^{\prime}\right) \text { and }\left(-\tau^{\prime},-\tau\right)
$$

are determined in $\mathfrak{D}_{0}^{*}$.

This result should be outlined at least well enough to reveal its elementary character: First of all, by (16.4) it clearly follows that

$$
\begin{array}{ll}
(e(\tau), e(\tau \sqrt{ } 3)) & \text { determines }\left(\tau, \tau^{\prime}\right)(\bmod 2), \\
\pm(e(\tau), e(\tau \sqrt{ } 3)) & \text { determines }\left(\tau, \tau^{\prime}\right)(\bmod 1+\sqrt{ } 3), \\
\pm\left(e(\tau), e\left(\tau \epsilon_{+}\right)\right) & \text {determines }\left(\tau, \tau^{\prime}\right)(\bmod 1+\sqrt{ } 3) \\
\pm\left(e(\tau), e\left(\tau \epsilon_{+}\right)+e\left(\tau \epsilon_{+}^{-1}\right)\right) & \text { determines }\left(\tau, \tau^{\prime}\right) \text { and }\left(-\tau^{\prime},-\tau\right) \\
& (\bmod 1+\sqrt{ } 3) .
\end{array}
$$

We now introduce new symbols

$$
E_{2}(\lambda \tau)=\sum_{-\infty}^{\infty} e\left(\lambda \tau \epsilon_{+}^{2 n}\right)
$$

so that

$$
E_{2}\left(\lambda \tau \epsilon_{+}^{2}\right)=E_{2}(\lambda \tau)
$$

Thus we rewrite (16.6) as

$$
\begin{aligned}
& \pm\left(E_{2}(\tau), E_{2}\left(\tau \epsilon_{+}\right)\right) \text {determines } \\
& \left(\epsilon_{+}^{2 n} \tau, \epsilon_{+}^{-2 n} \tau^{\prime}\right) \text { and }\left(-\epsilon_{+}^{2 n} \tau^{\prime},-\epsilon_{+}^{-2 n} \tau\right)(\bmod 1+\sqrt{ } 3) . \\
& \pm\left(E_{2}(\tau), E_{2}\left(\tau \epsilon_{+}\right)\right) \text {and } \pm\left(E_{2}\left(\tau \epsilon_{+}\right), E_{2}(\tau)\right) \text { determines } \\
& \quad\left(\epsilon_{+}^{n} \tau, \epsilon_{+}^{-n} \tau^{\prime}\right) \text { and }\left(-\epsilon_{+}^{2 n} \tau^{\prime},-\epsilon_{+}^{-2 n} \tau\right)(\bmod 1+\sqrt{ } 3) .
\end{aligned}
$$


Now we note that the pair $\left(A^{2}+B^{2}, A B\right)$ determines four pairs $\pm(A, B)$ and $\pm(B, A)$. On the other hand,

$$
\begin{aligned}
& \zeta_{1}=E_{2}^{2}(\tau)+E_{2}^{2}\left(\tau \epsilon_{+}\right)+\cdots, \\
& \zeta_{2}=E_{2}(\tau) E_{2}\left(\tau \epsilon_{+}\right)+\cdots
\end{aligned}
$$

Therefore, finally we have what we want:

$\left(\zeta_{1}, \zeta_{2}\right)$ determines the aggregate

$$
\begin{aligned}
& \left(\epsilon_{+}^{n} \tau+\nu(1+\sqrt{ } 3), \epsilon_{+}^{-n} \tau^{\prime}+\nu^{\prime}(1-\sqrt{ } 3)\right), \\
& \left(-\epsilon^{n} \tau^{\prime}+\nu(1+\sqrt{ } 3),-\epsilon_{+}^{-n} \tau+\nu^{\prime}(1-\sqrt{ } 3)\right)
\end{aligned}
$$

where $\nu \in \mathfrak{D}$. This aggregate is the set of $\tau$ which project into a given $\tau$ "modulo $\hat{\mathfrak{S}}_{6}^{*}$, " i.e., the set of transformations congruent $\bmod (1+\sqrt{ } 3)$ to $\tau \rightarrow \tau$ or $\tau \rightarrow-\tau^{\prime}$.

We therefore artificially extend the group $\mathfrak{S}_{6}^{*}$ to $\hat{\mathfrak{S}}_{6}^{*}$ with the adjunction of the symmetry operation

$$
\tau \rightarrow-\tau^{\prime}, \quad \tau^{\prime} \rightarrow-\tau .
$$

We call the completion $\bar{D}_{6}^{*}$ the neighborhood of $\left(\zeta_{1}, \zeta_{2}\right)=(0,0)$ or equivalently $\left(J_{1}, J_{2}\right)=(0,0)$.

Thus the only functions definable on the manifold $\overline{\mathfrak{D}}_{6}^{*}$ are then symmetric $G\left(\tau, \tau^{\prime}\right)=G\left(-\tau^{\prime},-\tau\right)$. This is clearly the case when the Fourier coefficients $A(\nu)=A\left(\nu^{\prime}\right)$ for conjugates, but for some forms, the symmetry can conceivably fail (although no cases are available).

Obviously this is as unsatisfactory as compactifying the Riemann sphere and discovering only functions which map reals into reals are definable.

A reasonable subject of curiosity would be the question of whether or not the functions $J_{1}, J_{2}$ provide a (symmetric) mapping of the "completed" $\left(\tau, \tau^{\prime}\right)$ domain $\overline{\mathfrak{D}}_{6}^{*}$ onto the bicomplex $J_{1}, J_{2}$ planes, with a two fold covering. (The property is valid at $\infty$ as we have seen.)

The study of nonsymmetric parameters has not yet been occasioned by a number theoretic property. Nevertheless the point can now be made that number theory is a tool of bicomplex analysis.

\section{BIBLIOGRAPHY}

1. H. Cohn, Decomposition into four integral squares in the fields of $2^{1 / 2}$ and $3^{1 / 2}$, Amer. J. Math. 82 (1960), 301-322.

2. - Calculation of class numbers by decomposition into three integral squares in the fields of $2^{1 / 2}$ and 31/2 Amer. J. Math. 83 (1961), 33-56. 
3. - Cusp forms arising from Hilbert's modular functions for the field of $3^{1 / 2}$, Amer. J. Math. 84 (1962), 283-305.

4. $H$. Cohn and G. Pall, Sum of four squares in a quadratic ring, Trans. Amer. Math Soc. 105 (1962), 536-556.

5. H. Cohn, On theta-functions for certain quadratic fields, Acta Arithmetica 9 (1964), 53-66.

6. - On the shape of the fundamental domain of the Hilbert modular group, Theory of Numbers, Proc. Sympos. Pure Math., Vol. 8, Providence, R. I., 1965, pp. 190-202.

7. - A numerical survey of the floors of various Hilbert fundamental domains, Math. Comp. 19 (1965), 594-605.

8. C. F. Gauss, Anziehung eines elliptischen Ringes (1818), Ostwald's Klassiker der exakten Wissenschaften 225 (1927).

9. F. Götzky, Über eine zahlentheoretische Anwendung von Modulfunktionen zweier Verändlichen, Math. Ann. 100 (1928), 411-437.

10. K. B. Gundlach, Some new results in the theory of Hilbert's modular group, Contributions to Function Theory, Bombay, 1960; pp. 165-188.

11. - Die Bestimmung der Funktionen zur Hilbertschen Modulgruppe des Zahlkörpers $Q(\sqrt{ } 5)$, Math. Ann. 152 (1963), 226-256.

12. - Die Fixpunkte einiger Hilbertscher Modulgruppen, Math. Ann. 157 (1965), 369-390.

13. E. Hecke, Analytische Funktionen und algebraische Zahlen. II, Abh. Math. Sem. Univ. Hamburg 3 (1924), 213-236.

14. H. Maass, Modulformen und quadratische Formen über dem quadratischen Zahlkoerper $R\left(5^{1 / 2}\right)$, Math. Ann. 117 (1941), 65-84.

15. - Quadratische Formen über quadratischen Körpern, Math. Z. 51 (1945), 233-254.

16. R. A. Rankin, Elliptic modular functions and forms, Lecture Notes, University of Indiana, Bloomington, Ind., 1964.

17. C. L. Siegel, Über die analytische Theorie der quadratischen Formen, Ann. of Math. 36 (1935), 527-606, ibid. 37 (1936), 230-263, ibid. 38 (1937), 212-291.

UnIVERSITY OF ARIzona 